description of Mantispa. It measures 20mm. in length.

\section{OCCURRENCE OF RARE MANTISPA SP. (NEUROPTERA: MANTISPIDAE) FROM TADOBA-ANDHARI TIGER RESERVE, MAHARASHTRA}

\author{
R.M. Sharma and S.S. Talmale \\ Zoological Survey of India, Western Regional Station, Vidyanagar, \\ Sector 29, PCNTD post office, P.Box No.3053, Akurdi, Pune, \\ Maharashtra 411044, India.
}

While collecting insects attracted to light on 20 March 1999 at Tadoba-Andhari Tiger Reserve, District Chandrapur, Maharashtra, we encountered a single specimen of genus Mantispa, a very rare insect of family Mantispidae of order Neuroptera.

Incidentally, a perusal of literature revealed no record of Mantispids from Maharashtra State. Thus, this note is intended to place on record the hitherto unrecorded, rare and interesting group of predatory insects. The rarity of the Mantispids from India is evident from the recent publication of Ghosh (1998) wherein he mentions that out of about 335 known neuropteran species under 125 genera spread over 13 families, Mantispids are represented by only four genera and 12 species. Worldwide, this family contains about 15 genera and 250 species (Henry, 1982). The specimen we collected agrees well with the general
The members of the family Mantispidae are commonly known as 'False Mantids' as they closely resemble true Mantids. These are small to medium-sized, soft and slender bodied insects, readily recongnised by their large hemispherical eyes, short antennae, elongate prothorax and the large raptorial forelegs as in mantids, thus commonly mistaken for those peculiar predators. Each femur is armed with powerful spines and the tibia is adapted to fold closely on to it; the two parts forming a very effective prehensile organ for seizing the prey. Their posterior legs are fit for walking. The two parts of hyaline wings are similar and rather narrow. The venation resembles somewhat that of Chrysopids (Green Lace wings). The adults are very rare and have never been found in great numbers. They occur in various types of vegetation where they simply wait for prey to reach within striking distance of their raptorial fore legs.

Further intensive surveys will certainly unearth the diversity of these obscure insects. While doing so proper attention should also be paid to conserve these precious creatures.

\section{Acknowledgement}

We are thankful to Dr. J.R.B. Alfred, Director, Zoological Survey of India, Calcutta and Dr. M.S. Pradhan, Scientist 'SE' and Officerin-Charge Z.S.I., W.R.S., Pune for facilities and encouragement.

\section{References}

Ghosh, S.K. (1998). Neuroptera. In: Faunal Diversity of India. (Eds: Alfred et.al.) pp. 252-257. ENVIS Centre Z.S.I., Calcutta.

Henry, C.S. (1982). Neuroptera. In: Synopsis and classification of living organisms. (Ed. Parker, S.P.), pp. 470-482, McGraw Hill Book Company, New York.

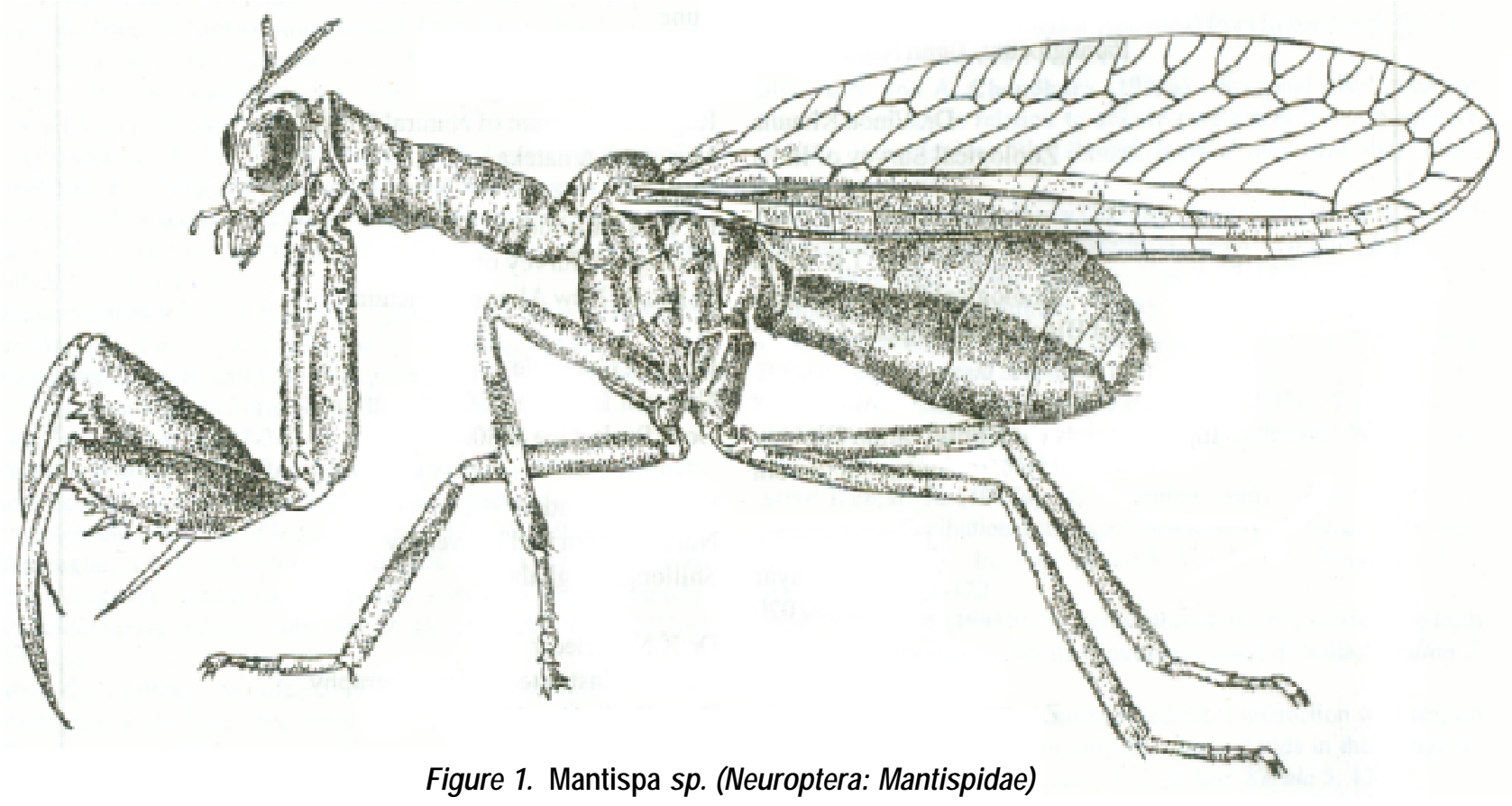

Received 3 July 1999;

Accepted 3 December 1999 